\title{
Attachment disorganization and controlling behavior in middle childhood: maternal and child precursors and correlates
}

\section{Citation}

Bureau, Jean François, M. Ann Easlerbrooks, and Karlen Lyons-Ruth. 2009. "Attachment Disorganization and Controlling Behavior in Middle Childhood: Maternal and Child Precursors and Correlates." Attachment \& Human Development 11 (3) (May): 265-284. doi:10.1080/14616730902814788.

\section{Published Version}

doi:10.1080/14616730902814788

\section{Permanent link}

http://nrs.harvard.edu/urn-3:HUL.InstRepos:37140322

\section{Terms of Use}

This article was downloaded from Harvard University's DASH repository, and is made available under the terms and conditions applicable to Other Posted Material, as set forth at http:// nrs.harvard.edu/urn-3:HUL.InstRepos:dash.current.terms-of-use\#LAA

\section{Share Your Story}

The Harvard community has made this article openly available.

Please share how this access benefits you. Submit a story.

Accessibility 


\title{
Attachment disorganization and controlling behavior in middle childhood: maternal and child precursors and correlates
}

\author{
Jean François Bureau ${ }^{a,}{ }^{*}$, M. Ann Easlerbrooks ${ }^{b}$, and Karlen Lyons-Ruth ${ }^{c}$ \\ aUniversity of Ottawa \\ bTufts University \\ 'Harvard Medical School
}

\section{Abstract}

Main, Kaplan, and Cassidy's (1985) conceptualizations of disorganization in infancy and controlling behavior in preschool forged new directions in attachment research. However, there currently is no valid coding system for behavioral manifestations of attachment after 7 years of age. The present study presents the validity of an instrument for coding both disorganized and rolereversed behavior from ages 7 to 9; the Middle Childhood Disorganization and Control (MCDC) scales. In the present study, 43 mother-child dyads at socioeconomic risk, followed from infancy, were assessed on the MCDC at age 8. Analyses revealed that children with higher combined punitive/disorganized scores were significantly more likely to: (1) come from families referred for clinical home visiting because of concerns about the quality of the parent-infant relationships; (2) have higher scores for disorganization in infancy if from the clinically-referred subgroup; (3) are more likely to show disorganized representations; and (4) show higher externalizing and internalizing behavior problems. Higher caregiving MCDC scores were associated with more maternal withdrawal behavior in infancy. In conclusion, the present study provided the first validity data for an observational measure of disorganization and control in middle childhood.

\section{Keywords}

disorganized attachment; controlling attachment; role-reversal; middle childhood; at-risk; parentchild interaction

\section{Introduction}

Longitudinal research on attachment beyond infancy has documented that disorganized behavior in infancy may, over time, become organized as controlling patterns of attachment, as preschoolers may attempt to control parental behavior in a caregiving or punitive fashion (Main \& Cassidy, 1988; Wartner, Grossmann, Fremmer-Bombik, \& Suess, 1994). We do not know, however, how attachment disorganization may be manifest in older children. 
Currently there is no valid system for coding behavioral manifestations of attachment disorganization after 7 years of age; this is despite the fact that one might anticipate changes in the markers of attachment disorganization beyond early childhood, given increasingly sophisticated communicative, perspective-taking and cognitive capacities. The present study aims to assess the validity of an instrument for coding aspects of attachment disorganization in middle childhood (age 8).

Infants are viewed as developing disorganized attachment behaviors through exposure to the caregiver's frightening or frightened behavior (Hesse \& Main, 2006) and/or through the caregiver's unavailability to respond to infant fears or distress from other sources (LyonsRuth, Bronfman, \& Parsons, 1999). Lyons-Ruth, Bronfman, and Parsons (1999) developed the AMBIANCE coding system for assessing such disrupted caregiver responses to infant affective cues when under stress. This system allows coding for a variety of forms of disrupted responses to infant cues, including role confusion, disorientation, intrusiveness and hostility, affective communication errors and withdrawal. Four studies $(N=384)$ have now shown associations between disrupted maternal communication as assessed on the AMBIANCE scales and disorganized attachment in infancy, with a meta-analytic effect size of $r=.35$ (Madigan, Bakermans-Kranenburg, van IJzendoorn, Moran, Pederson, \& Moran, 2006). Lyons-Ruth, Bronfman, and Atwood (1999) also found more specific patterns of association between maternal withdrawal and the disorganized/secure classification and between maternal hostile-intrusiveness and the disorganized/insecure classification. Finally, results from recent studies (see Lyons-Ruth \& Jacobvitz, 2008, for a review) suggest that genetic predisposition may moderate the association between the quality of parenting and child disorganization.

These associations between disrupted caregiving and infant disorganization are also consistent with results from the meta-analytic study of van IJzendoorn, Schuengel, and Bakermans-Kranenburg (1999) showing that the proportion of disorganized attachments is dramatically higher among abusive or neglecting families (see also Cicchetti \& Barnett, 1991). It is important to note that the same meta-analytic study indicated a nonsignificant association between disorganized attachment behavior in infancy and constitutional and temperamental variables $(r=.003)$. Child gender was also unrelated to disorganized attachment in metaanalyses (van IJzendoorn et al., 1999).

As disorganized infants and toddlers make the transition into the preschool years, the signs of conflict, apprehension, or helplessness characteristic of disorganized attachment strategies in infancy often give way to various forms of controlling behavior toward the parent. This developmental shift toward an increasing proportion of controlling behavior patterns with the parent over the preschool years has been documented in follow-up studies at age 6 of three middle-income samples (Main \& Cassidy, 1988; Moss, Cyr, Bureau, Tarabulsy, \& Dubois-Comtois, 2005; Wartner et al., 1994). The controlling behaviors are manifest in either a caregiving or a punitive fashion. Children who are classified as controlling-punitive use "authoritarian" behavior with the caregiver that may include harsh commands, verbal threats, and occasional physical aggression toward the parent. Children classified as controlling-caregiving direct the parent's activities and conversational exchanges by structuring interactions in a helpful and/or emotionally positive manner. Solomon, George, 
and De Jong (1995) suggested that controlling behaviors function to control a parent who is the source of unintegrated fears, thereby regulating the child's own internal state and behavior. It has been suggested that these two profiles may be associated with different risk factors, with the child's caregiving behavior associated with more helpless and passive maternal behavior, and the child's punitive behavior associated with more hostile and intrusive maternal behavior (Lyons-Ruth, Lyubchik, Wolfe, \& Bronfman, 2002; Solomon \& George, 2008).

Further results from Moss, Cyr, and Dubois-Comtois (2004) found that the controllingcaregiving pattern was associated with a history of loss in the family. This association was also confirmed by Solomon and George (2007) who described an unusually high rate of complicated bereavement reported by mothers of controlling-caregiving children. These authors also showed that the internal working models of caregiving children are marked by inhibition and anxiety while the models of punitive children are characterized by representations of chaos and violence (Solomon et al., 1995). Finally, their results showed that some controlling children were described by their mothers as being wild and acting out, while others were described as being precocious and caregiving (George \& Solomon, 1996).

It is important to note that this apparent transformation from a disorganized to a controlling attachment pattern does not occur for all children with disorganized attachments. Studies show that $25-33 \%$ of disorganized infants do not adopt a controlling attachment pattern by age 6 (see Moss et al., 2005). Moreover, it has been shown that in higher-risk samples the proportion of disorganized infants who develop controlling strategies by the end of the preschool period is smaller than in low-risk samples (Cicchetti \& Barnett, 1991). Hence, there is considerable heterogeneity in the attachment behavior associated with disorganization by school entry.

While many disorganized attachments may become transformed into organized strategies over time, developmental sequelae of disorganization in infancy are also characteristic of controlling children. Longitudinal studies have documented that by preschool age, disorganized infants display a higher incidence of both disruptive behavior and internalizing symptoms of depression and anxiety than do children with organized strategies in infancy (see DeKlyen \& Greenberg, 2008, for a review). Studies of preschool and early school age attachment show similar findings, with associations also present between controllingdisorganized attachment behaviors and externalizing and/or internalizing behavior problems (for reviews, see Lyons-Ruth \& Jacobvitz, 2008; Moss, Bureau, St-Laurent, \& Tarabulsy, 2009). Recent work by Moss and her colleagues (Moss et al., 2004) suggests more specific associations between a controlling-punitive profile and externalizing behavior and a controlling-caregiving profile and internalizing problems. As noted earlier, these findings have all been generated using the coding systems for controlling and disorganized behavior validated up to age 7 . However, no reliable measures of disorganized and controlling behaviors have been validated for children older than 7 years, and criteria for disorganization or control at earlier ages may not be appropriate for the older child.

The present study aims to validate a new measure of disorganized and controlling behavior of 8-year-old children evaluated in a reunion with their mothers (Middle Childhood 
Disorganization and Control scales; Bureau, Easterbrooks, Killam, \& Lyons-Ruth, 2006) adapted from the preschool coding systems developed by Cassidy and Marvin (1992) and Main and Cassidy (1988). Based on theory and extant literature on attachment disorganization, the Middle Childhood Disorganization and Controlling (MCDC) scales describe three dimensions of children's behavior toward their caregivers: controllingpunitive, controlling-caregiving, and disorganized behavior. The focus of the current coding system is on expression of disorganization and controlling behavior rather than on the traditional attachment classifications (secure, avoidant, ambivalent-resistant), because our interest was in forms of attachment that may be associated with psychopathology.

\section{Validity assessment}

The validity of the MCDC scales will be explored following a model based on Solomon and George's (2008) suggestions concerning the validation process of attachment constructs. Merging Nunnally's (1978) model with a more traditional model, Solomon and George proposed to assess the different dimensions as part of a validation process. In line with this model we will first explore the reliability of the measure (i.e., whether codes requiring judgment be agreed upon). Second, we will explore the construct validity of the system. As part of the construct validity, we will examine the long-term stability, the convergence with another measure of disorganization in middle childhood, and the associations with core variables. Solomon and George (2008) proposed two categories of core variables that should be related to an attachment construct: quality of parenting and social adaptation.

\section{Objectives and hypotheses}

Following the model proposed by Solomon and George, we explored the following aspects of instrument validity. First, the reliability of the scales was evaluated by assessing interjudge reliability; we expected good interjudge reliability for each of the three scales. Second, the construct validity was examined. It was expected that higher scores on the MCDC scales would be associated with: (1) higher scores of disorganization of attachment measured in infancy; (2) a higher rate of disorganized representations in childhood; (3) the presence of early clinical risk; (4) higher rates of behavior problems as reported by mothers; and (5) the presence of maternal disrupted communication in infancy. More specifically, Lyons-Ruth and colleagues (Lyons-Ruth et al., 2002) have proposed that maternal helplessness and withdrawal in infancy will be associated with the emergence of child caregiving behaviors while maternal hostility and intrusiveness will predict child punitive behaviors. Therefore, those differential predictions were also tested.

\section{Method}

\section{Participants}

The data presented in this study were collected through mother-child laboratory visits when children were infants (age $M=18.17$ months, $S D=2.29$, range from 12 to 24 months; $n=$ 76 ), and school aged (age $M=8.56$ years, $S D=.47$, range from 7.3 to 9.6 years, $n=43$ ). Forty-three mother-child dyads ( 24 male, 19 female children) were seen in a lab visit when children were 8 years old. 
Families were recruited from a larger cohort of families followed from infancy. The infant sample consisted of 76 infant-mother dyads that were assessed for maternal disrupted communication and infant attachment security. Of those dyads, 41 (18 girls) were lowincome families referred to the study by health or social service agency staff because of concerns about the quality of the parent-infant relationship. Thirty-five ( 15 girls) were families from the community demographically matched to referred families on the following seven factors: per-person family income; mother's education, age, and race; and infant's age, sex, and birth order. Community families were screened for documented psychiatric histories, history of abuse or neglect of children, or obvious parenting difficulties during a 1hour home observation. To participate in the study, all families were required to be below the official federal poverty level. In infancy, 66\% of families had incomes under US\$50 per person/per week, and $62 \%$ received government aid. The sample was $81 \%$ Caucasian, $11 \%$ Latino, 4\% African American, and 4\% biracial children. Forty percent of mothers were not high school graduates and $49 \%$ were single parents.

At the 8-year follow-up, five of the original 76 families refused participation, and the remainder of the drop-out group could not be relocated. The families who participated in the study in middle childhood did not differ significantly from those who did not participate on demographic measures in infancy such as family income, $F(1,75)=.98$, n.s., maternal education, $\chi^{2}(76)=.32$, n.s., ethnicity, $\chi^{2}(76)=.87$, n.s., and single mother family, $\chi^{2}(76)$ $=.55$, n.s. However there was a significant difference between the two groups based on clinical risk, $\chi^{2}(76)=4.53, p<.05$. A higher proportion of families referred for parentinfant clinical services did not come back for the 8-year-old follow-up (54\% versus $30 \%$ of comparison families). Attrition in middle childhood was not related to any of the infancy period observations: attachment disorganization, $F(1,70)=1.75$, n.s., or maternal disrupted communication, $F(1,64)=3.26$, n.s.

\section{Procedure}

When children were 8-year-old, their interaction with their mothers was observed in a separation-reunion procedure. Dyads were separated for approximately an hour. Maternal reports about the child were collected during the separation. Child receptive language intelligence was collected in the laboratory 1 year prior to this visit.

\section{Clinical risk}

A score of 1 on clinical risk was assigned to the participants referred for clinical home visiting and a score of 0 was assigned to the non-referred group. These participants were presented earlier as being referred because of concerns about the quality of parent-infant relationship.

\section{Infant attachment disorganization (Infancy)}

Mothers and infants were videotaped in the Strange Situation Procedure (Ainsworth, Blehar, Waters, \& Wall, 1978) between 12 and 18 months of age. In this procedure the infant is videotaped in a playroom during a series of eight structured 3-minute episodes involving the baby, the mother, and a female stranger. During the observation the mother leaves and rejoins the infant twice, first leaving the infant with the female stranger, then leaving the 
infant alone. The procedure is designed to be mildly stressful in order to activate the infant's attachment behavioral system. Videotapes were coded for the three infant attachment classifications as described by Ainsworth et al. (1978) and for disorganized/disoriented behaviors as described by Main and Solomon (1990). The three original attachment classifications (secure, avoidant, ambivalent) were assigned by both a computerized multivariate classification procedure developed on the original Ainsworth data (Connell, 1976; see also reference in Richters, Waters, \& Vaughn, 1988) and a coder trained by M. Main. Agreement between the two sets of classifications on the full infancy data set was $86 \%$. Infants were observed for disorganized/disoriented behaviors in the Strange Situation as described by Main and Solomon (1990). A continuous ninepoint scale yielded scores ranging from 1 to 5 increasing by half-points (e.g. 1.5, 2.0, 2.5); scores of 4 or 5 classified the child as disorganized (the original version of the disorganized scale developed by Main and Solomon). Agreement on the nine-point Level of Disorganized Behavior Scale between M. Main and another coder for 32 randomly selected tapes was $r=.84$. Given the large proportion of disorganization in the sample ( $43 \%$ of the sample) and the study focus on disorganization, the major attachment variable for this study was the extent of disorganization scale. The use of a continuous measure of extent of disorganization was used to maximize the power of the analyses.

\section{Disrupted maternal affective communication (Infancy)}

Disrupted maternal communication with the infant was coded using the AMBIANCE coding system (Lyons-Ruth, Bronfman, \& Parsons, 1999) based on behavior over all episodes of the Strange Situation Procedure. Coders of the videotapes were naïve to the criteria for coding disorganized attachment behavior as well as all other data on the sample. The AMBIANCE coding protocol yields a scaled score (1-7) for extent of disrupted communication, with five subtotals for frequency of affective communication errors, role confused behavior, negative-intrusive behavior, fearful/disoriented behavior, and withdrawal. Fifteen randomly selected tapes were coded by two coders to assess reliability. The reliabilities were as follows: Level of Disrupted Communication Scale, $\kappa=.93$; Affective Communication Errors Subscore, $r_{i}=.75$; Role Confusion Subscore, $r_{i}=.76$; Negative-Intrusive Behavior Subscore, $r_{i}=.84$; Disorientation Subscore, $r_{i}=.73$; Withdrawal Subscore, $r_{i}=.73$.

\section{Child verbal IQ (Age 7)}

Child receptive verbal intelligence at age 7 was evaluated using the well-validated Peabody Picture Vocabulary Test-Revised (PPVT-R; Dunn \& Dunn, 1981). This test of receptive language skills gives a standardized score with a mean of 100 and a standard deviation of 15 for a normative population. Previous studies have shown that PPVT-R scores are significantly correlated with more extensive measures of IQ such as the WWPSI, WISC and WAIS (see Carvajal, Hayes, Miller, \& Wiebe, 1993).

\section{Middle Childhood Disorganization and Control (MCDC) scales (Age 8)}

The MCDC scales (range from 1 to 9) rate the extent of three dimensions of children's behavior toward their parent: controlling-punitive, controlling-caregiving, and disorganized 
behavior coded in a separation-reunion laboratory setting. The interactions coded for the present study were observed in a 5-minute reunion following a 1-hour separation during which both child and mother were interviewed by examiners. Behavior in the low range of the controlling-punitive scale expresses frustration, annoyance, or impatience toward the parent. The high range of this scale is characterized by episodes of hostility toward the parent that are marked by a challenging, humiliating, cruel or defying quality. The low range of the controlling-caregiving scale includes minor indications of caregiving behavior with the motivation of stimulating, modifying affect, or distracting the parent. The high range of the scale is marked by a more active form of organizing or taking charge of the interaction. Evidence of the child subordinating his/her own desires and prioritizing the parent's needs are also coded on this scale. For both of these scales, a score of 1 is assigned to the child who doesn't express controlling behavior. The disorganized behavior scale includes the following categories of behavior in the presence of the parent: (1) manifestations of fear; (2) lack of a consistent interaction strategy (e.g. child shows caregiving behaviors following a period of extreme avoidance); (3) unpredictable, confused behavior when approaching the parent at the moment of reunion or when reconnecting with the parent after an argument or prolonged avoidance; (4) behavior that invades the parent's intimate boundaries (e.g. trying to kiss mother on the mouth despite her protest); (5) difficulties in expression when addressing the parent, in absence of speech difficulties in other circumstances; (6) extreme self-negating comments or self-injurious behaviors; (7) markers of disorientation or dissociation; and (8) clear preference for the stranger. Each behavior coded in one of these categories is categorized as either low or high. The combination of low and high disorganized behavior scores leads to an overall rating of disorganization on a 1-9 scale. A score of 1 is assigned to a child who shows no signs of disorganization.

The scales were developed based on observations of 99 episodes (reunions and free play sessions) of 44 mother-child dyads from three additional independent samples (low-risk, $n=$ 19; mixed-economic risk, $n=11$; and high-risk [documented history of abuse or neglect], $n$ =14). All children from the development samples were between 7 and 9 years old. The scales developed on these observations were then applied to the current study sample.

Interrater reliability was calculated on $51 \%(n=22)$ of the current study sample. Intraclass correlations for the three MCDC scales were as follows: Punitive $=.97$; Caregiving $=.93$; and Disorganization $=.83$. In order to explore the interrelations among the three scales, a factor analysis with varimax rotation was conducted. An eigenvalue higher than 1.00 was used as a criterion for factorial retention. The analysis showed that a first factor (eigenvalue $=1.40$ ) included the punitive and the disorganization scales. A second factor (eigenvalue = 1.02) included the caregiving scale exclusively. The correlation between the punitive and disorganized scales was moderate $(r=.30)^{1}$, while the caregiving scale was negatively related to the punitive scale $(r=-.28)$ and was not associated with the disorganized scale $(r$ $=.02$ ). For this reason, a composite (mean) score representing punitive and disorganized scales and one score representing the caregiving scale were used in subsequent analyses.

\footnotetext{
${ }^{1}$ Note that the correlation between the punitive and the disorganized scales is very similar when controlling for age (partial $r=.25$ ).
} 
However, considering that this is a new instrument, results for individual scales are also presented for descriptive purposes.

\section{Representations of attachment (Age 8)}

The Separation Anxiety Test (SAT; Kaplan, 1987) was administered in order to assess children's representations of child-mother attachment security. The SAT consists of six line-drawings (same age/same gender child as participant child) depicting child-mother separations of various intensity (e.g. mother goes out for the evening, mother puts child to bed, mother goes away for 2 weeks). The child is asked: (1) how the pictured child feels and (2) what the pictured child is going to do. Responses were classified into attachment categories according to criteria developed by Chazan (1995), based on the work by Main and her colleagues (1985). A secure classification was assigned when a child maintained a balance between self-exposure and self-containment, admitted vulnerability in terms of negative feelings and generated positive coping responses. An avoidant classification was assigned when a child showed little sense of personal vulnerability and denied negative feelings. An ambivalent classification was assigned when a child exhibit strong distress and vulnerability without generating adaptive coping responses. Finally, a disorganized classification was assigned when a child demonstrated a lack of regulation, both emotionally and behaviorally. Responses might be fearful, extreme or bizarre (e.g. jump out the window, burn down the house) or coping behaviors that increased the distance between the child and attachment figure (e.g. child says the mother will go away for 4 weeks instead of 2 weeks). SAT vignettes were scored from videotape and transcript by four trained coders, who first trained on a sample of SAT transcripts provided by Kaplan (1987). Two coders classified each SAT, with a Cohen's kappa of .92 for the four attachment classifications (secure, avoidant, ambivalent, and disorganized). Reliability and validity of the Kaplan's coding system for the SAT was very good in previous studies (Kaplan, 1987; see also Solomon \& George, 2008, for a review).

\section{Mother report of child behavior problems (Age 8)}

When children were age 8, data were collected on mothers' reports of children's behavior problems with the Child Behavior Checklist (CBCL; Achenbach \& Edelbrock, 1983). This instrument consists of 118 items scored on a three-point scale (not true, somewhat or sometimes true, very true or often true). The CBCL generates scores on 9 subscales; withdrawn, somatic complaints, anxious/depressed, social problems, thought problems, attention problems, delinquent behavior, aggressive behavior, and other problems. The addition of the delinquent behavior and aggressive behavior subscales yields an externalizing score, while the addition of the withdrawn, somatic complaints and anxious/ depressed subscales yields an internalizing score. Standardized $t$ scores for sex and age were used in this study. The instrument has excellent psychometric qualities. Test-retest reliability was established for short-term ( 7 days; average of .89 for all scales) as well as for long-term (average of .75 after 1 year and .71 after 2 years for all scales). Analyses reveal that almost all the $\mathrm{CBCL}$ items discriminate between clinically referred and non-referred children, indicating excellent construct validity. 


\section{Life events (Age 8)}

Mothers were asked to report the presence (1) or absence (0) of a series of life events that could have occurred during a period of 3 years prior to the lab visit at age 8 . For the purpose of the present study, the different events were regrouped in terms of their themes. The groups are the following: (1) illness/death of a family member; (2) abortion or miscarriage; (3) problems at school; (4) changes in work status or problems at work (mother); (5) parents' separation or departure of a family member; (6) family violence or problems in the family; (7) financial or legal problems; (8) moving or difficulty with housing; and (9) a new person in the family (new child or new partner).

\section{Results}

\section{Preliminary analyses}

Due to the uniformly low-income nature of the sample in infancy, preliminary analyses did not reveal any significant relations of family income or single parenthood with either of the MCDC factors. Pun-D factor: income, $r=-.02$, n.s.; single, $F(1,42)=.18$, n.s. (single parent: $m=2.19, S D=1.75, n=21$; two parents: $m=2.41, S D=1.59, n=22)$. Punitive scale: income, $r=.01$, n.s.; single, $F(1,42)=.04$, n.s. (single parent: $m=2.48, S D=2.14, n$ $=21$; two parents: $m=2.36, S D=1.40, n=22$ ). Disorganized scale: income, $r=-.06$, n.s.; single, $F(1,42)=.07$, n.s. (single parent: $m=1.67, S D=1.32, n=21$; two parents: $m=1.77$, $S D=1.23, n=22)$. Caregiving scale: income, $r=-.27$, n.s.; single, $F(1,42)=.13$, n.s. (single parent: $m=3.29, S D=1.87, n=21$; two parents: $m=3.09, S D=1.72, n=22$ ). Analyses of associations between age of the child and MCDC scales revealed a significant association between age and combined Pun-D score only, $r=-.33, p=.03$, with older children showing less controlling/ disorganized behaviors. Due to this significant result, age was controlled in subsequent analyses. Descriptive statistics for the principal continuous study variables are presented in Table 1.

\section{Associations with child gender}

There were no significant differences between boys and girls on the combined Pun-D scale, $F(1,42)=.02$, n.s. (girls: $m=2.26, S D=1.63, n=19$; boys: $m=2.33, S D=1.71, n=24$ ); the punitive scale, $F(1,42)=.01$, n.s. (girls: $m=2.42, S D=1.71, n=19$; boys: $m=2.42, S D$ $=1.86, n=24)$; the caregiving scale, $F(1,42)=.01$, n.s. (girls: $m=3.21, S D=2.07, n=19$; boys: $m=3.17, S D=1.55, n=24$ ); or the disorganized scale, $F(1,42)=.03$, n.s. (girls: $m=$ 1.68, $S D=1.25, n=19$; boys: $m=1.75, S D=1.29, n=24)$. Therefore, consistent with preschool data, girls were equally likely to display punitive forms of interaction as were boys, and boys were as likely as girls to display caregiving behavior.

\section{Associations with child IQ}

As expected, child IQ scores assessed with the PPVT-R (Dunn \& Dunn, 1981) a year earlier were not associated with the combined Pun-D scale, $r=-.20$, n.s.; the punitive scale, $r=-$. 03 , n.s.; the caregiving scale, $r=-.16$, n.s.; or the disorganization scale, $r=-.16$, n.s. Based on this lack of association, neither child gender nor child IQ was controlled in the subsequent analyses. 


\section{Construct validity of the MCDC scales}

Long-term stability-Zero-order correlations between the continuous score for infant disorganization and the MCDC scales in middle childhood did not reach significance, with Pun-D scale $r=.29$, n.s., disorganization scale $r=.23$, n.s., punitive scale $r=.24$, n.s., and caregiving scale $r=-.25$, n.s., although the correlation of .29 with the combined Pun-D scale was sizeable. Based on the growing literature suggesting that stability of attachment is quite different in low and high parenting risk samples (see Moss et al., 2005, for a review), a multiple regression analysis was also conducted to test a possible moderation effect of clinical risk on the concordance between infant disorganization and the MCDC scales in middle childhood. Analyses revealed a significant interaction between clinical risk and infant disorganization in the prediction of the combined Pun-D MCDC scores (see Table 2). Further exploration of this significant interaction effect indicated a strong and significant correlation between infant disorganization and the combined Pun-D score 7 years later in the clinical group, $r=.53, p=.03$, but no association in the non-clinical group, $r=.09$, n.s.

Given this moderation of stability by clinical risk, other potential moderators of stability were also examined. One additional series of analyses explored whether the stability of disorganization differed as a function of life events occurring in middle childhood (between 5 and 8 years of age). Moderation analyses, similar to those described for clinical risk, were run for each of the nine groups of life events for each of the four MCDC scales. Only one significant moderation effect was revealed. A new work status or problem at work (mother) moderated the relation between infant disorganization and caregiving behavior toward the parent in middle childhood (disorganization $\times$ work status, $\beta=1.24, p=.05$ ). Follow-up analysis of the interaction term showed that infant disorganization was negatively associated with caregiving behavior in the context of stable work status or no experience of work problems $(r=-.55, n=23)$. However, infant disorganization was positively associated with caregiving behavior at age 8 when mothers reported a change in work status or experiencing problems at work ( $r=.24, n=17)$. Finally, it is interesting to note that, although they did not moderate stability of disorganization, both financial/legal problems and violence and problems in the family between 5 and 8 were correlated with higher ratings on the disorganized scale in middle childhood, $r=.38$ and $r=.34$, respectively.

A second set of analyses explored whether stability of disorganization differed as a function of disrupted maternal communication in infancy. Neither the overall level of disruption scale nor any of the five subscales were significant moderators of the association between infant disorganization and the middle childhood disorganization and control scales.

Convergence with a concurrent measure of disorganization-Concerning the association between the child's representations of attachment at age 8 and disorganized and controlling behavior at age 8 , a series of simple contrasts was run in order to compare the scores of children classified with disorganized attachment representations on the SAT to children with each of the other classifications (secure, avoidant, or ambivalent). These analyses revealed that children classified disorganized on the SAT had higher scores on the combined Pun-D scale and on the Disorganized scale than did children who had secure, avoidant, or ambivalent representations on the SAT. The disorganized children on the SAT 
also had higher punitive scores alone than did secure children. Means, standard deviations, and significant contrasts are presented in Table 3.

Association with core variables: clinical risk-Univariate analyses of covariance (ANCOVA) were used to explore the difference between referred and non-referred groups on the MCDC scale scores. Results revealed that children in the referred group $(m=2.24$, $S D=1.64, n=17$ ) obtained significantly higher disorganized scale scores than did children in the non-referred group $(m=1.38, S D=.80, n=26), F(1,42)=5.15, p=.02$. Children in the referred group ( $m=2.88, S D=1.96, n=17$ ) also obtained marginally higher combined Pun-D scores than children in the non-referred group ( $m=1.92, S D=1.32, n=26), F(1,42)$ $=3.67, p=.06$. Analyses did not reveal significant effects of clinical risk status on the punitive, $F(1,42)=.11$, n.s. (referred: $m=2.53, S D=1.59, n=17$; non-referred: $m=2.35$, $S D=1.92, n=26$ ), or caregiving scales $F(1,42)=.10$, n.s. (referred: $m=3.29, S D=1.72, n$ = 17; non-referred: $m=3.12, S D=1.84, n=26$ ).

Association with core variables: maternal disrupted communication-Multiple regression analyses were conducted to test three a priori hypotheses concerning disrupted maternal communication in infancy (Lyons-Ruth, Bronfman, \& Atwood, 1999) and later disorganized/controlling behavior on the MCDC scales. First, overall maternal disrupted communication was expected to predict overall levels of disorganized/controlling behavior in middle childhood. A series of independent regression analyses revealed that, when controlling for child age and other MCDC scales, a higher score for overall level of maternal disrupted communication in infancy was particularly associated with the child's punitive score at age $8, \Delta F(1,29)=5.88, p<.05$. No significant associations were found for the combined Pun-D score, $\Delta F(1,29)=3.27$, n.s.; disorganized score, $\Delta F(1,29)=.13$, n.s.; or caregiving score, $\Delta F(1,29)=1.74$, n.s., scores.

In addition, Lyons-Ruth et al. (2002) had theorized that hostile maternal stances in infancy would predict later child punitive control while helpless maternal stances would elicit child caregiving behavior over time. Consistent with these predictions, maternal withdrawal in infancy significantly predicted child caregiving at age 8 , with age and other scales controlled, $\Delta F(1,29)=6.16, p<.05$. Contrary to prediction, the maternal negative/intrusive score alone did not predict child punitive behavior at age $8, \Delta F(1,29)=.81$, n.s., in contrast to the significant prediction of punitive behavior from the broader set of disrupted behaviors demonstrated above.

Associations with core variables: child behavior problems-A series of regressions analyses was conducted to explore the associations between the total behavior problem scores of the mother-reported CBCL (Achenbach \& Edelbrock, 1983) and the MCDC scales (see Table 4). Regression analyses revealed that, when controlling for child age, clinical risk and other MCDC scales, both disorganized MCDC subscales and combined Pun-D MCDC scales significantly predicted higher mother-reported behavior problems at age 8. Further multiple regression analyses were run to explore whether MCDC subscales were associated with both externalizing and internalizing behavior problems as reported by mothers (see Table 4). Results showed that the combined Pun-D scale significantly predicted both higher externalizing and higher internalizing scores, after controlling for age, clinical 
risk, and otherMCDC scales. Similarly, the MCDC scale for disorganization significantly predicted both higher externalizing and higher internalizing scores, after controlling for age, clinical risk, and other MCDC scales. As presented in Table 4, the caregiving scale was not significantly associated with any of the behavior problem scores.

\section{Discussion}

The objective of the present study was to explore the validity of an instrument evaluating school-age children's disorganized and controlling behavior toward their mothers in a separation-reunion procedure. Validity was explored following a model proposed by Solomon and George (2008), including exploring the reliability and construct validity of the instrument. First, reliability of the instrument was documented by high intercoder intraclass correlations for each of the scales. Second, in relation to construct validity, we hypothesized that disorganized and controlling behavior as assessed on the MCDC scales would be associated with disorganized attachment behavior measured in infancy and with disorganized representations of attachment measured concurrently at age 8. Results partially supported these hypotheses. The longitudinal association between infant disorganization and the combined punitive-disorganization scale at age 8 was sizeable $(r=.29)$ but failed to reach significance. However, when moderated by clinical risk, a large effect size emerged $(r$ $=.55)$ for those in the clinical group. Concurrent representations of attachment disorganization on the SAT at age 8 were also significantly related to punitive and disorganized behavior, independently and in combination. Finally, we expected that higher scores on the middle childhood disorganization and controlling scales would show retrodictive relations with disrupted maternal affective communication and clinical risk measured in infancy and would also be linked to concurrent child behavior problems at 8 years old. Early clinical risk was specifically related to later disorganization, rather than to controlling behavior, while different forms of early maternal behavior predicted the two different forms of controlling behavior, as discussed below.

Developed based on previous literature on infant disorganization and preschool attachment, the MCDC scales were developed to include indicators of punitive, caregiving, and disorganized behavior. Preliminary analyses showed coherence among punitive and disorganized behavior, while caregiving behavior was not associated with either punitive or disorganized behavior. These data support the significance of utilizing a coding system that allows examination of the extent of each of these three aspects of disorganized/controlling behavior at age 8 . Published studies examining post-infancy attachment behavior previously have employed classification systems which hinder exploration of the associations between different subtypes of disorganized/controlling behavior. Continuous scales should allow greater precision in the evaluation of more sophisticated and subtle displays of behavior when compared to a dichotomous classification of behavior as dysfunctional (disorganized or controlling) or functional (organized and non-controlling). The use of independent scales also allows one to capture the degree to which the different subtypes of disorganized/ controlling behavior are or are not commingled.

Our data showed a strong association between indicators of fear and anxiety captured by the disorganization scale and punitive, though not caregiving, behavior. We suggest that an 
organized caregiving strategy is manifest in a tendency to show a super-competent self who is able to take care of the dyad regardless of the behavior of the parent. For these children, demonstrations of fear, anxiety, or disorganization might indicate an intolerable admission of vulnerability that could threaten the confidence the parent could put in the child's caregiving capacities. The implication is not that these children are not anxious; rather, that their expressions of anxiety may be manifest in behaviors that do not signal vulnerability to the parent (e.g. overbright greeting). This hypothesis remains to be verified but is consistent with results obtained by Solomon et al. (1995) showing that, when participating in a dollplay narrative task, caregiving children are generally constricted and refuse to tell stories that could potentially expose their vulnerability or their anger.

We predicted that clinical levels of risk in the caregiving environment during infancy would be associated with children's behavior as assessed by the MCDC scales; this hypothesis was supported by links with both the disorganized scale and, marginally, the combined punitivedisorganized scale. Such an association is consistent with attachment theory. The lack of a link between clinical risk and later child caregiving behavior may be due, in part, to the fact that this strategy requires the child to hide or contain frustration and vulnerability, a strategy that may be more difficult to organize in a more adverse environment. Thus, we propose that a caregiving strategy fails to organize, or partially breaks down, with an accumulation of risk factors, giving way either to the demonstrations of frustration, aggression, and domineering control associated with punitive behavior, or to the fear, confusion, and disorientation associated with disorganized behavior. Future studies should explore this hypothesis further.

A meta-analysis of predominantly low-risk samples (van IJzendoorn et al., 1999) has shown stability in the disorganized classification from infancy to preschool age, including the transformation of the infant disorganized classification to preschool controlling attachment. Based on these results, it was expected that infant disorganization would predict the childhood scales. This prediction was partially supported. Results obtained in the present study indicated that infant disorganization was strongly associated with the combined disorganized-punitive scale in middle childhood, but only in the clinical group. This suggests that additional risk factors are necessary to catalyze the continuation of disorganized behavior into school age. It is possible that some disorganized children in lessdisturbed family settings rely on the new relationships available over time (e.g. teachers and friends) in order to improve their self-regulation skills and this may be reflected in their interaction with their mothers at 8 years old. However, disorganized children living in abusive or neglecting families may experience more difficulty establishing such compensatory relationships. Thus, they may maintain disorganized behavioral strategies, which would explain a higher stability of disorganization in a high-risk context.

It is also interesting to note that the association between infant disorganization and caregiving behaviors in middle childhood differed as a function of the work status and/or problems experienced at work by mothers. One may conjecture that a mother who gets a new job or experiences problems at work may be less available, thus explicitly or implicitly asking her child to assume more responsibilities. The transition from disorganization to caregiving behavior may not be related to abuse or hostility, but rather to the physical or 
emotional availability of the mother. This finding needs to be replicated, however, given the lack of specific a priori predictions and the large number of tests conducted.

The construct validity of the scales was also supported by the associations shown between classifications of disorganization on the SAT, a representational measure of attachment and punitive and disorganized interaction with the mother assessed on the MCDC. Slough and Greenberg (1990) also found an association between their SAT security scales assessed in 5year-olds and attachment behaviors observed in a separation-reunion procedure. Solomon et al. (1995) also demonstrated correspondence between controlling behavior at age 6 and concurrent representations of fear and disorganization in doll-play. This study extends those prior findings into middle childhood. It is important to note however that only four children were classified as disorganized on the basis of the SAT in the present study.

Maternal disrupted communication in infancy predicted child punitive and caregiving behavior 7 years later. More specifically, overall maternal disrupted communication predicted child punitive behavior, while maternal withdrawal in infancy predicted child caregiving behavior. The association between maternal withdrawal and child caregiving behavior is consistent with the prediction of Lyons-Ruth et al.(2002) that infants of more withdrawn mothers would be more likely to develop caregiving behaviors, based on their continued attempts to approach their mothers in infancy (see David \& Lyons-Ruth, 2005). Moss et al. (2009) also observed that mothers of caregiving preschoolers often appeared quite passive and disengaged, showing a neutral or negative affective expression. In addition, George and Solomon (1996) suggested that mothers of controlling-caregiving children may paradoxically abdicate at the level of behavior (e.g. cease to make effort or take interest in the mother-child relationship), while at the same time feeling exceptionally close to their children, with an emphasis on the children's precociousness.

The last objective of the present study was to explore the association between the middle childhood scales of attachment disorganization and children's behavior problems. Moss and her colleagues (reviewed in Moss et al., 2009) found that punitive and disorganized preschool attachment behavior with the parent was associated with externalizing symptoms while caregiving behavior toward the parent tended to be related to internalizing problems in middle childhood (rated by both teachers and mothers). In the present sample, disorganized behavior, alone and in combination with punitive behavior, predicted both externalizing and internalizing behavior problems reported by mothers. Consistent with other results obtained in this study, children combining punitive and disorganized features were exhibiting the greatest difficulties in social adaptation.

The lack of association between the punitive scale alone and externalizing problems was unexpected. While not explaining the lack of association, it may indicate that this scale is not simply a measure of general aggression, especially since usual markers on the lower end of the scale are more subtle displays of impatience or annoyance such as rolling eyes or refusing to answer a question. These behaviors are not necessarily related to physical or verbal aggression measured in externalizing behavior problems scales. However, it should be noted that mothers were the reporters of behavior problems and there is a possibility that 
mothers of punitive children might tend to overlook the aggressive behaviors of their children. Teacher ratings are needed to assess this possibility.

The lack of association between the caregiving scale and internalizing behavior also was unexpected. It is important to note that Moss et al. (2004) found that preschool-aged children classified as caregiving were differentiated only from the secure children on internalizing behaviors; they were not different from children in any other attachment groups, including controlling-punitive and disorganized. In another study, Moss and her colleagues questioned maternal reports of internalizing problems and recommended using self-reports of internalizing symptoms with school-aged children (Moss, Smolla, Cyr, Dubois-Comtois, Mazzarello, \& Berthiaume, 2006).

It may also be that as children move into middle childhood, the less severely at risk children are able to organize a controlling strategy, as well as to conform to the demands of the social environment, so that only those who continue to exhibit disorganized behavior will also exhibit behavior problems. Short-term longitudinal studies from kindergarten to middle childhood are needed to assess these potential developmental changes. Moreover, as discussed previously, the caregiving strategy and its correlates have not been studied under conditions of high social risk. Previous work by Moss and her colleagues linking caregiving classifications to negative outcomes was conducted with mostly well-functioning families and may not be generalizable to high risk environments and/or to assessments in middle childhood. In the current study, by age 8 , children's caregiving behavior was not associated with increased clinical risk or with infant disorganized behavior. However, the scale showed the previously predicted association with maternal withdrawal in infancy. It is possible that, in the face of maternal withdrawal, a caregiving strategy can only be organized and sustained by more competent infants and children (e.g. NICHD Early Child Care Research Network, 2001). Alternately, David and Lyons-Ruth (2005) found that maternal withdrawal evoked approach behavior in infancy, so that some infants of very withdrawing parents were classified secure rather than disorganized. Therefore, disturbed attachment in infancy may be less likely to be detected in this subgroup (see Lyons-Ruth et al., 2002).

The study has several limitations that should be noted. First, the design included a relatively small number of participants studied over a long period of time ( 8 years), which diminished the power of the analyses. In addition, the nature of the sample (families at socio-economic risk) limits the generalization of the results to samples at lower risk. The high risk nature of the sample also could partly explain the high attrition level from infancy to 8 years old. It will be important to replicate these results in different samples with different socioeconomic characteristics. Nonetheless, the study design presented a unique opportunity to explore, for the first time, disorganized and controlling behaviors in middle childhood among families at high social risk.

Finally, many aspects of the validity of the middle childhood scales remain to be explored. First, the short-term stability and long-term predictive validity of the scales should be explored. Second, the measurement of other variables such as child temperament would be useful in establishing the discriminant validity of the scales. Third, construct validity could be further explored in relation to measures of maternal behavior at age 8 , home 
observations, and behavior problems reported by teachers. Finally, the validity of the scales would benefit from studies conducted in different cultural contexts. Nonetheless, this first study presents encouraging results on a newly created instrument. It is our hope that these results will stimulate an interest in using the scales with different populations of children in middle childhood, both in research and clinical settings.

Largely neglected until recently (Kerns \& Richardson, 2005), research done at school age has used a variety of representational measures for assessing children's attachment strategies, including doll-play narratives (Bretherton, Ridgeway, \& Cassidy, 1990; Oppenheim, 1997; Solomon et al., 1995), the Separation Anxiety Test (Kaplan, 1987), or questionnaires (Kerns, Klepac, \& Cole, 1996). In addition, there have been recent attempts at using "AAI-format" interviews with school-age children (Ammaniti, Speranza, \& Fedele, 2005; Target, Fonagy, \& Shmueli-Goetz, 2003). However, validated observational assessments for this age range have been notably absent. In addition, there is still no evidence that representational assessments reflect the observed organization of attachment relationships. These behavioral scales could fill this gap in attachment research and could be useful in linking earlier behavioral measures (Cassidy \& Marvin, 1992;Main \& Cassidy, 1988) with an understanding of adaptation among school-age children.

\section{References}

Achenbach, TM.; Edelbrock, CS. Manual for the Child Behavior Checklist and Revised Child Behavior Profile. Burlington, VT: Department of Psychiatry, University of Vermont; 1983.

Ainsworth, MDS.; Blehar, MC.; Waters, E.; Wall, S. Patterns of attachment: A psychological study of the strange situation. Hillsdale, NJ: Erlbaum; 1978.

Ammaniti, M.; Speranza, AM.; Fedele, S. Attachment in infancy and in early and late childhood. In: Kerns, KA.; Richardson, RA., editors. Attachment in middle childhood. New York: Guilford Press; 2005. p. 115-136.

Bretherton, I.; Ridgeway, D.; Cassidy, J. Assessing internal working models of the attachment relationship: An Attachment Story Completion Task for 3-year-olds. In: Greenberg, MT.; Cichetti, D.; Cummings, EM., editors. Attachment in the preschool years: Theory, research, and intervention. Chicago: University of Chicago Press; 1990. p. 3-49.

Bureau, J-F.; Easterbrooks, MA.; Killam, S.; Lyons-Ruth, K. Middle Childhood Disorganization and Controlling Scales. Cambridge: Unpublished coding manual, Harvard Medical School; 2006.

Carvajal H, Hayes JE, Miller HR, Wiebe DA. Comparisons of the vocabulary scores and IQs on the Wechsler Intelligence Scale for Children-III and the Peabody Picture Vocabulary Test-Revised. Perceptual and Motor Skills. 1993; 76:28-30.

Cassidy, J.; Marvin, RS. with the McArthur Working Group on Attachment. Attachment organization in $2 \frac{1}{2}$ to $4 \frac{1}{2}$ year olds. Coding manual. Charlottesville: Unpublished coding manual, University of Virginia; 1992.

Chazan, RZ. Attachment and self-concept in high risk school age sample. Medford, MA: Unpublished master's thesis, Tufts University; 1995.

Cicchetti D, Barnett D. Attachment organization in maltreated preschoolers. Development and Psychopathology. 1991; 3:397-411.

Connell, DB. Individual differences in attachment: An investigation into stability, implications, and relationships to structure of early language development. New York: Unpublished doctoral dissertation, Syracuse University; 1976.

David DH, Lyons-Ruth K. Differential attachment responses of male and female infants to frightening maternal behavior: tend or befriend versus fight or flight? Infant Mental Health Journal. 2005; 26:1-18. [PubMed: 17464362] 
DeKlyen, M.; Greenberg, MT. Attachment and psychopathology in childhood. In: Cassidy, J.; Shaver, PR., editors. Handbook of attachment: theory, research, and clinical applications. 2nd ed.. New York: Guilford Press; 2008. p. 637-665.

Dunn, LM.; Dunn, LM. Peabody Picture Vocabulary Test-Revised. Circle Pines, MN: American Guidance Service; 1981.

George C, Solomon J. Representational models of relationships: Links between caregiving and attachment. Infant Mental Health Journal. 1996; 17:198-216.

Hesse E, Main M. Frightened, threatening, and dissociative parental behavior in low-risk samples: Description, discussion, and interpretations. Development and Psychopathology. 2006; 18:309343. [PubMed: 16600057]

Kaplan, N. Individual differences in six-year-olds' thoughts about separation: Predicted from attachment to mother at one year of age. University of California at Berkeley: Unpublished doctoral dissertation; 1987.

Kerns KA, Klepac L, Cole AK. Peer relationships and preadolescents' perceptions of security in the child-mother relationship. Developmental Psychology. 1996; 32:457-466.

Kerns, KA.; Richardson, RA. Attachment in middle childhood. New York: Guilford Press; 2005.

Lyons-Ruth, K.; Bronfman, E.; Atwood, G. A relational diathesis model of hostile-helpless states of mind: Expressions in mother-infant interaction. In: Solomon, J.; George, C., editors. Attachment disorganization. New York: Guilford Press; 1999. p. 33-70.

Lyons-Ruth K, Bronfman E, Parsons E. Vondra J, Barnett D. Maternal disrupted affective communication, maternal frightened or frightening behavior, and disorganized infant attachment strategies. Atypical patterns of infant attachment: theory, research and current directions. Monographs of the Society for Research in Child Development. 1999; 64(3):67-96.

Lyons-Ruth, K.; Jacobvitz, D. Attachment disorganization: Genetic factors, parenting contexts, and developmental transformation from infancy to adulthood. In: Cassidy, J.; Shaver, PR., editors. Handbook of attachment: theory, research, and clinical applications. 2nd ed.. New York: Guilford Press; 2008. p. 666-697.

Lyons-Ruth, K.; Lyubchik, A.; Wolfe, R.; Bronfman, E. Parental depression and child attachment: Hostile and helpless profiles of parent and child behavior among families at risk. In: Goodman, S.; Gotlib, I., editors. Children of depressed parents: Alternative pathways to risk for psychopathology. Washington, DC: American Psychological Association Press; 2002. p. 89-121.

Madigan S, Bakermans-Kranenburg MJ, van IJzendoorn MH, Moran G, Pederson DR, Benoit D. Unresolved states of mind, anomalous parental behavior, and disorganized attachment: A review and meta-analysis of a transmission gap. Attachment \& Human Development. 2006; 8:89-111. [PubMed: 16818417]

Main M, Cassidy J. Categories of response to reunion with the parent at age six: Predictable from infant attachment classifications and stable over a 1-month period. Developmental Psychology. 1988; 24:415-526.

Main, M.; Kaplan, N.; Cassidy, J. Security in infancy, childhood, and adulthood: A move to the representational level. In: Bretherton, I.; Waters, E., editors. Growing points of attachment theory and research. Vol. 50. Monographs of the Society for Research in Child Development; 1985. p. 66-104.

Main, M.; Solomon, J. Procedure for identifying infants as disorganized/disoriented during the Ainsworth Strange Situation. In: Greenberg, MT.; Cicchetti, D.; Cummings, EM., editors. Attachment in the preschool years: Theory, research, and intervention. Chicago: University of Chicago Press; 1990. p. 121-160.

Moss, E.; Bureau, J-F.; St-Laurent, D.; Tarabulsy, GM. Understanding disorganized attachment at preschool and school age: Examining divergent pathways of disorganized and controlling children. In: Solomon, J.; George, C., editors. Attachment disorganization. 2nd ed.. New York: Guilford; 2009. (in press).

Moss E, Cyr C, Bureau J-F, Tarabulsy G, Dubois-Comtois K. Stability of attachment between preschool and early school-age and factors contributing to continuity/discontinuity. Developmental Psychology. 2005; 41:773-783. [PubMed: 16173874] 
Moss E, Cyr C, Dubois-Comtois K. Attachment at early school age and developmental risk: Examining family contexts and behavior problems of controllingcaregiving, controlling-punitive, and behaviorally disorganized children. Developmental Psychology. 2004; 40:519-532. [PubMed: 15238040]

Moss E, Smolla N, Cyr C, Dubois-Comtois K, Mazzarello T, Berthiaume C. Attachment and behavior problems in middle childhood as reported by adult and child informants. Development and Psychopathology. 2006; 18:425-444. [PubMed: 16600062]

NICHD Early Child Care Research Network. Child-care and family predictors of preschool attachment and stability from infancy. Developmental Psychology. 2001; 37:847-862. [PubMed: 11699758]

Nunnally, JC. Psychometric theory. New York: McGraw-Hill; 1978.

Oppenheim D. The attachment doll-play interview for preschoolers. International Journal of Behavioral Development. 1997; 20(4):681-697.

Richters JE, Waters E, Vaughn BE. Empirical classification of infant-mother relationships from interactive behavior and crying during reunion. Child Development. 1988; 59:512-522. [PubMed: 3359869]

Slough N, Greenberg MT. Five-year-olds' representations of separation from parents: Responses from the perspectives of self and other. New Directions for Child Development. 1990; 48:67-84. [PubMed: 2216012]

Solomon, J.; George, C. Intergenerational patterns of representation and interaction: Threat, helplessness, and controlling patterns in mother and child. Symposium presented at the Society for Research in Child Development biennial meeting; Boston, MA. 2007.

Solomon, J.; George, C. The measurement of attachment security and related constructs in infancy and early childhood. In: Cassidy, J.; Shaver, PR., editors. Handbook of attachment: theory, research, and clinical applications. 2nd ed.. New York: Guilford Press; 2008. p. 383-416.

Solomon J, George C, De Jong A. Children classified as controlling at age six: Evidence of disorganized representational strategies and aggression at home and at school. Development and Psychopathology. 1995; 7:447-464.

Target M, Fonagy P, Shmueli-Goetz Y. Attachment representations in schoolage children: The development of the child attachment interview (CAI). Journal of Child Psychotherapy. 2003; 29:171-186.

van IJzendoorn MH, Schuengel C, Bakermans-Kranenburg MJ. Disorganized attachment in early childhood: Meta-analysis of precursors, concomitants, and sequelae. Development and Psychopathology. 1999; 11:225-250. [PubMed: 16506532]

Wartner UG, Grossman K, Fremmer-Bombik E, Suess G. Attachment patterns at age six in South Germany: Predictability from infancy and implications for preschool behavior. Child Development. 1994; 65:1014-1027. 


\section{Table 1}

Descriptive statistics for the principal continuous study variables.

\begin{tabular}{lrrc}
\hline Variable & Mean & Standard deviation & Range \\
\hline Disorganized infancy scale & 3.36 & 1.16 & $1-5$ \\
Maternal disrupted communication (infancy) & 4.00 & 1.74 & $1-7$ \\
Maternal withdrawal (infancy) & 2.74 & 3.06 & $0-11$ \\
Maternal hostility (infancy) & 2.03 & 3.33 & $0-14$ \\
Child IQ (age 7) & 101.86 & 16.89 & $52-137$ \\
Punitive scale (age 8) & 2.42 & 1.78 & $1-7$ \\
Caregiving scale (age 8) & 3.19 & 1.78 & $1-7$ \\
Disorganized scale (age 8) & 1.72 & 1.26 & $1-5$ \\
Pun-D combined scale (age 8) & 2.30 & 1.66 & $1-6$ \\
CBCL-total (age 8) & 53.36 & 8.99 & $34-72$ \\
CBCL-internalizing (age 8) & 60.28 & 10.17 & $40-80$ \\
CBCL externalizing (age 8) & 59.28 & 10.73 & $38-85$ \\
\hline
\end{tabular}




\section{Table 2}

Infant disorganization and clinical risk as predictors of combined Pun-D MCDC scale.

\begin{tabular}{lcccc}
\hline Variable & $\Delta \boldsymbol{R}^{2}$ & $\Delta \boldsymbol{F}$ & $d f$ & $\boldsymbol{\beta}$ \\
\hline Step 1 & .11 & $4.84^{*}$ & 1,41 & \\
Child age & & & & $-.33^{*}$ \\
Step 2 & .15 & $3.92^{*}$ & 2,39 & \\
Infant disorganization & & & & .19 \\
Clinical risk & & & & $.31^{*}$ \\
Step 3 & .09 & $5.09^{*}$ & 1,38 & \\
D-infancy X clinical risk & & & & $1.47^{*}$ \\
\hline$*$ & & & & \\
$p<.05$. & & & &
\end{tabular}




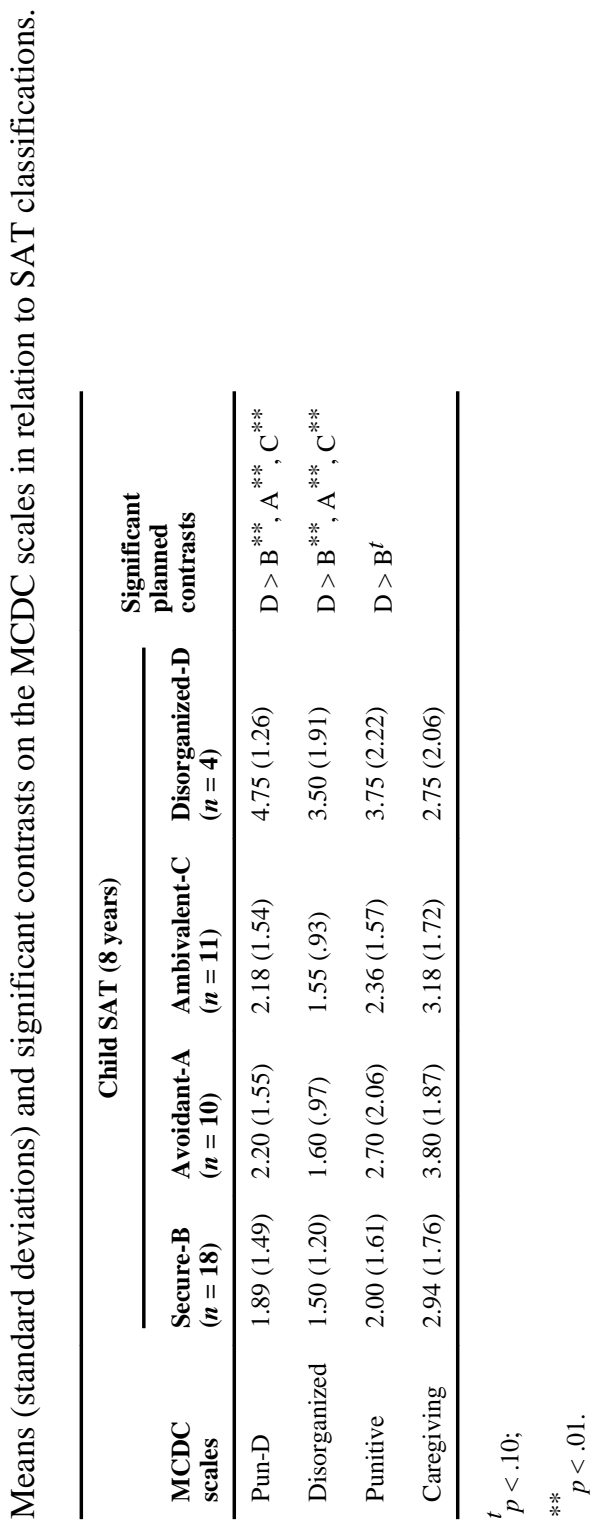




\section{Table 4}

MCDC scales as predictors of total behavior problems as reported by mothers.

\begin{tabular}{lcccc}
\hline Variable $^{\boldsymbol{a}}$ & $\Delta \boldsymbol{R}^{2}$ & $\Delta \boldsymbol{F}$ & $d f$ & $\beta$ \\
\hline Total behavior problems & & & & \\
Model 1. Pun-D & .21 & $11.35^{* *}$ & 1,34 & $.51^{* *}$ \\
Model 2. Disorganized & .31 & $19.85^{* *}$ & 1,34 & $.61^{* *}$ \\
Model 3. Punitive & .01 & .24 & 1,34 & .08 \\
Model 4. Caregiving & .01 & .14 & 1,34 & .06 \\
Externalizing behavior problems & & & & \\
Model 1. Pun-D & .14 & $7.66^{* *}$ & 1,34 & $.42^{* *}$ \\
Model 2. Disorganized & .23 & $14.89^{* *}$ & 1,34 & $.53^{* *}$ \\
Model 3. Punitive & .01 & .04 & 1,34 & -.03 \\
Model 4. Caregiving & .02 & .68 & 1,34 & .12 \\
Internalizing behavior problems & & & & \\
Model 1. Pun-D & .20 & $8.65^{* *}$ & 1,34 & $.49^{* *}$ \\
Model 2. Disorganized & .25 & $11.83^{* *}$ & 1,34 & $.55^{* *}$ \\
Model 3. Punitive & .02 & .52 & 1,34 & .13 \\
Model 4. Caregiving & .01 & .09 & 1,34 & -.05 \\
\hline
\end{tabular}

${ }^{a}$ Age, clinical risk, and other MCDC scales were controlled for in each of the regression models.

* $p<.05 ;$

$* *$ p $<.01$. 